\title{
Neural Mechanisms Underlying the Impact of Visual Distraction on Retrieval of Long-Term Memory
}

\author{
Peter E. Wais, Michael T. Rubens, Jacqueline Boccanfuso, and Adam Gazzaley \\ Departments of Neurology, Physiology, and Psychiatry, W. M. Keck Center for Integrative Neurosciences, University of California, San Francisco, \\ San Francisco, California 94158-2330
}

Filtering information on the basis of what is relevant to accomplish our goals is a critical process supporting optimal cognitive performance. However, it is not known whether exposure to irrelevant environmental stimuli impairs our ability to accurately retrieve longterm memories. We hypothesized that visual processing of irrelevant visual information would interfere with mental visualization engaged during recall of the details of a prior experience, despite goals to direct full attention to the retrieval task. In the current study, we compared performance on a cued-recall test of previously studied visual items when participants' eyes were closed to performance when their eyes were open and irrelevant visual stimuli were presented. A behavioral experiment revealed that recollection of episodic details was diminished in the presence of the irrelevant information. A functional magnetic resonance imaging experiment using the same paradigm replicated the behavioral results and found that diminished recollection was associated with the disruption of functional connectivity in a network involving the left inferior frontal gyrus, hippocampus and visual association cortex. Network connectivity supported recollection of contextual details based on visual imagery when eyes were closed, but declined in the presence of irrelevant visual information. We conclude that bottom-up influences from irrelevant visual information interfere with top-down selection of episodic details mediated by a capacity-limited frontal control region, resulting in impaired recollection.

\section{Introduction}

Environmental stimulation inundates our senses and creates a constant demand for cognitive control processes to inhibit the internalization of information that is irrelevant to our goals. Accordingly, the integrity of higher-order cognition is dependent upon the suppression of such interference, as shown for working memory and perception (Rainer et al., 1998; Lavie et al., 2004; Zanto and Gazzaley, 2009). Yet, despite the established importance of filtering distraction for optimal cognitive performance, it is not known whether irrelevant external stimulation interferes with the retrieval of long-term memories. In the current study, we first explored the behavioral consequences of exposure to irrelevant visual information on recollection performance, and then investigated the neural basis of interference effects in a functional magnetic resonance imaging (fMRI) experiment.

Long-term memory (LTM) of episodic information, or recollection, is an effortful process that retrieves more elaborate memories than simple recognition (Atkinson and Juola, 1973; Mandler, 1980). Psychological models propose that recollection uses visual imagery of the details of prior episodes (James, 1890; Tulving, 1985). Neural evidence of mental imagery evoked during LTM has been investigated using fMRI, and these studies have

Received March 23, 2010; accepted April 23, 2010.

This project was supported by National Institutes of Health Grant R01-AG30395 and the American Federation of Aging. We thank C. Brock Kirwan for making the unique stimuli available.

Correspondence should be addressed to Adam Gazzaley, UCSF-MC2512, 1600 16th Street, Room N474, San Francisco, CA 94158-2330. E-mail: adam.gazzaley@ucsf.edu.

DOI:10.1523/JNEUROSCI.1478-10.2010

Copyright $\odot 2010$ the authors $\quad 0270-6474 / 10 / 308541-10 \$ 15.00 / 0$ revealed that subjective recollection involves the reinstatement of sensory cortical activity associated with the encoding of a prior event (Buckner and Wheeler, 2001; Wheeler and Buckner, 2004; Johnson and Rugg, 2007; Johnson et al., 2009). For example, visual imagery during LTM is supported by activity in stimulusselective areas in the visual association cortex (Ishai et al., 2000; Kahn et al., 2004; Mechelli et al., 2004; Ranganath et al., 2004; Wheeler and Buckner, 2004; Johnson and Rugg, 2007; Stokes et al., 2009). Recollection also depends upon memory areas in the medial temporal lobe (MTL) (Squire et al., 2007) and cognitive control contributions from the prefrontal cortex (PFC) (Badre and Wagner, 2007).

We hypothesized that because visual imagery in support of recollection uses the same limited-capacity buffers in the visual association cortex that are involved in processing external visual stimuli (de Fockert et al., 2001; Lavie, 2005), as well as overlapping cognitive control networks (Blumenfeld and Ranganath, 2006), visual stimulation during a retrieval effort disrupts the recollection of details about a prior experience. This may be related to common acts of looking away or closing one's eyes when engaged in effortful recollection (Glenberg et al., 1998), reflexive actions that may serve to block interference between irrelevant external information and recalling details from memory.

Previous behavioral studies have shown that engagement in a secondary cognitive task during LTM (i.e., divided attention) interferes with free recall (Fernandes and Moscovitch, 2000) and source memory (Troyer et al., 1999). The motivation for the current study was to investigate the impact of distraction by entirely irrelevant visual information on recollection. Our approach 
was to cue participants to recall previously studied objects during blocks when their eyes were closed, or were open and irrelevant visual information was present (Fig. 1). In an fMRI experiment we examined the neural networks that support recollection and visual imagery, and how functional connectivity between brain regions is impacted by the presence of irrelevant visual information.

\section{Materials and Methods}

\section{Behavioral experiment}

Participants. Twenty-nine university students (13 males), who were native speakers of English, gave their informed consent to perform the experimental tasks in return for course credit or a small fee. Two participants did not comply with the instructions, and their data were excluded from analysis.

Stimuli. One hundred sixty-eight object images, one image of a $25 \%$ gray screen, and 160 images of outdoor scenes were displayed on a computer screen at $1024 \times 768$ pixel resolution. Each object image displayed one to four copies of a common object from a three-dimensional perspective, in color, on a plain white background. The displacement in the viewable area from the objects was held as constant as possible, whereas the actual objects varied in size (i.e., wishbones versus sofas). The number of target images with each count of objects $(1,2,3$, or 4$)$ was equated. The outdoor scenes were a range of neutral pictures of complex natural scenes, street scenes, sculptures and nebulae. Stimuli were presented using E-prime 2.0 (Psychology Software Tools).

Procedure. The experiment investigated the impact of distraction by irrelevant visual information on recollection, and its design included neither an explicit, nor implicit secondary task. Our approach used a cued-recall procedure to assess recollection of episodic information. The critical manipulation was to test LTM of visual information during conditions when visual stimulation was nil (participants' eyes shut so that visual processing of external stimuli could not interfere with processes engaged during memory retrieval) and when visual environmental stimuli were presented concurrently with auditory recall cues (eyes open looking at pictures of complex scenes).

The experiment was separated into three sessions: encoding, test and posttest. Written instructions were read out loud to each participant by the experimenter before each session, and the participant then completed a brief practice run for each session with the experimenter.

During the encoding session, each of the 168 target images was presented for $3000 \mathrm{~ms}$, in random order, over two runs. When participants viewed each image during the first run, they entered a yes or no answer into the computer keyboard to indicate their judgment about whether one of the objects from the image could fit inside a lady's shoebox, and, during the second run, to indicate whether they believed they could carry all of the objects from the image across the room using only their hands and arms. Each of the 336 trials were preceded by a $2000 \mathrm{~ms}$ fixation cross, and $12 \mathrm{~s}$ rest periods occurred after each block of 56 trials.

After a $1 \mathrm{~h}$ break, participants were instructed about a surprise memory test. Six test blocks ( 34 trials in each block, 28 targets presented in a random order with 6 lures) were presented in one of three pseudorandom orders. Each trial began with a fixation cross on a white background for $2500 \mathrm{~ms}$, followed by an auditory cue that described an object encoded in the previous session, or a novel (i.e., unstudied) object, in singular form. Participants were instructed to recall the count for the object described by the cue from a study image and give their answer by pressing 1, 2, 3, 4 or "new" (pressing all four buttons simultaneously) on a response pad, as rapidly as possible without sacrificing accuracy. The 2500 $\mathrm{ms}$ test period was followed by a $10 \mathrm{~s}$ intertrial interval (ITI), the last second of which included a visual (enlarged fixation cross) or auditory cue (two beeps) to indicate the next trial was about to begin.

The participants gave recollection responses during three different conditions (i.e., two blocks in each condition): when visual stimulation was nil (eyes closed: SHUT), when bottom-up processing was minimal (looking at a gray screen: GRAY), and when neutral, visual environmental stimuli were presented (looking at pictures of scenes: Visual Distraction, or VD) (Fig. 1). The visual stimuli appeared simultaneously with the presentation of the auditory cue and remained on screen for $2500 \mathrm{~ms}$. Participants were instructed to fix their gaze at the center of the computer screen during stimulus presentation in GRAY and VD trials. On each trial in the SHUT condition, an auditory beep forewarned participants $1000 \mathrm{~ms}$ in advance of the auditory cue onset. Before each block during the GRAY and VD conditions, participants were instructed to hold their gaze constant on the center of the viewing screen throughout each trial and "not to blink or look away when the screen changes from the fixation cross." The sequence of test conditions (GRAY, VD, and SHUT) was pseudorandomized after the first test block, which was always GRAY in an effort to optimize orientation to the task across participants.

Correct responses for the object count indicated successful recollection. Incorrect object count for previously studied images revealed successful recognition, but failed recollection of the details of the study image. The trials in which the probe for a studied image was given a "new" response were classified as forgotten. Probes for unstudied images (i.e., lures) that were erroneously given an object count were classified as false alarms and that were given a "new" response were classified as correct rejections.

Immediately after the test session was completed, participants were given a surprise after test for their recognition of the Visual Distractors. In this third session, the 80 outdoor scenes from the VD test blocks and 80 thematically matched lures were presented in random order. On each trial, after a fixation appeared on a white background for $2000 \mathrm{~ms}$, each scene was presented for $2500 \mathrm{~ms}$ while participants gave an answer whether the scene was old or new with a confidence rating (i.e., $1=$ definitely new, 2 = probably new, $3=$ probably old, $4=$ definitely old). After the short retention interval, memory discrimination for the Visual Distractors showed mean $d^{\prime}=0.93 \pm 0.08$. At the conclusion of the three sessions, participants completed a verbal exit interview in which the experimenter inquired about the strategy adopted to solve the recall question and compliance with the instructions to not look away from the visual distractors. 


\section{fMRI experiment}

Participants. Twenty-five healthy adults (mean age $=23.3$ years $\pm 3.0,9$ males) recruited from the university community, gave consent and were monetarily compensated to participate in the study. Participants had normal or corrected-to-normal vision and were prescreened to exclude individuals using medication known to affect cognitive state. Two participants' data were excluded from the final analysis due to their failure to adhere to task instructions: one participant after the analysis of eyetracker data, and the other participant on the basis of exit-interview responses.

Stimuli. All of the images were the same as the behavioral experiment.

Procedure. The encoding and after-test sessions were conducted outside of the scanner and were the same as in the behavioral experiment. The test session, which began after participants were comfortably situated in the fMRI scanner with high-performance SereneSound headphones (Resonance Technology), included six memory test blocks, one Passive Viewing (PV) block (identical to the VD condition, expect there were no auditory cues), one independent Region of Interest Localization block described below in the fMRI data analysis, and an anatomical scan. Eye-tracking data (described below) were recorded during the six memory test blocks.

fMRI acquisition. All images were acquired on a Siemens 3T Magnetom Trio. Thirty-six $3.2 \mathrm{~mm}$ axial $\mathrm{T}^{\star}$-weighted gradient-echo slices $(1.8 \times 1.8 \times 3.2 \mathrm{~mm}$ voxel size, $0.5 \mathrm{~mm}$ interslice gap, $\mathrm{TR}=2500 \mathrm{~ms}$, $\mathrm{TE}=30 \mathrm{~ms}, 80^{\circ}$ flip angle, and $230 \mathrm{~mm}^{2} \mathrm{FoV}$ in a $128 \times 128$ matrix) were collected. Slice acquisition was aligned approximately with the longitudinal axis of the hippocampus so that coverage included the ventral extent of the temporal lobes (for two participants, coverage ended at the medial extent of the precentral gyrus). Images were corrected for slice timing, motion artifacts and smoothed with a $5 \mathrm{~mm}$ FWHM Gaussian. Data were modeled using a general linear model (GLM) in SPM5. Group whole-brain maps were calculated from Montreal Neurological Institute (MNI) normalized data. In addition, high-resolution anatomical (T1MPRAGE) data sets were collected.

fMRI data analysis. BOLD responses were modeled as brief epochs convolved with the canonical hemodynamic response function (HRF) in SPM5 (Wellcome Department of Cognitive Neurology, London, UK). The seven event-related fMRI sessions (two each for SHUT, GRAY and $\mathrm{VD}$, and one for PV) were concatenated within the GLM to increase the power of regressors with sparse onsets. The model included 10 taskrelated regressors based on the categorization of participants' responses as: correct recall of the count of target items during SHUT, GRAY or VD trials; incorrect count of target items during SHUT, GRAY or VD trials; all trials when targets were incorrectly endorsed as new (forgotten); all trials when lures were endorsed as old (false alarms); all trials when lures were correctly rejected (CR) as new; and all PV trials. Forgotten, false alarm and CR trials were collapsed across test conditions into respective regressors to make it possible to model these three categories of behavioral responses with few observations. Additionally, several nuisance variables were added to the model, including three translational $(X, Y, Z)$ and three rotational (pitch, roll, yaw) motion parameters, scan-specific linear drift regressors and scan block constants (i.e., baselines). BOLD time courses were generated from the average difference of the signal and baseline (intercept) across a region of interest (ROI) for each time point (TR). The percentage signal change (PS $\Delta$ ) was computed as: (signal baseline) $\times$ 100/baseline.

fMRI localizer task and analysis. The scanner session always began with an independent localizer task that was used to identify stimulus-selective regions of interest (ROI) in the visual association cortex. Participants performed a one-back task during three repetitions of $17.5 \mathrm{~s}$ blocks that presented 35 object stimuli similar to the targets, grid-scrambled object stimuli, or natural scene stimuli. Each stimulus block was separated by a $17.5 \mathrm{~s}$ rest period (fixation). Participants were instructed to indicate when an image matched the preceding image within a block with a button press. Based on the conclusions from Schwarzlose et al. (2008), two contrasts were made to generate $\mathrm{SPM}[\mathrm{T}]$ images for the identification of ROIs: objects $>$ grid-scrambled objects to localize object-selective areas; and scenes $>$ objects to localize scene-selective areas. The specific ROIs represent object-selective areas in the lateral occipital cortex (LOC) and scene-selective areas in the parahippocampal place area (PPA).

$f M R I$ univariate analysis. Evaluation of the neural basis of interference effects involved first analyzing univariate whole-brain data using an ANOVA that compared $\beta$-values associated with three factors: hits (i.e., SHUT correct, SHUT incorrect, VD Correct and VD incorrect); misses (i.e., targets that were forgotten); and correct rejections. Identification of regions that exhibited significant main effects (corrected for multiple comparisons) was followed by a priori pairwise comparisons, which enabled the localization of general memory-related ROIs. These pairwise comparisons adopted the more conservative approach of applying SEs of the respective mean differences, rather than the overall ANOVA error term (Anderson, 2001). ROIs were then further evaluated with a second ANOVA to address our primary hypothesis of an impact of distraction on recollection via detecting an interaction of two factors: recollection performance (correct vs incorrect) and condition (SHUT vs VD) (corrected for multiple ROI comparisons). These regions were then used as seed ROIs in a functional connectivity analysis.

fMRI functional connectivity analysis. The $\beta$-series-correlation method was used to analyze whole-brain functional connectivity using seed ROIs obtained by group statistical maps in MNI space, which were then warped into native space for each participant. This transformation was carried out using the parameters derived from warping the SPM EPI template to match the average EPI across each subject's first functional run. For this analysis, a new GLM design matrix was constructed to model each trial with a unique covariate, resulting in a total of 238 covariates of interest ( 34 trials per session $\times 7$ sessions). Whole-brain maps of functional connectivity were generated for each participant by extracting $\beta$ values for each trial from each seed ROI and correlating the mean $\beta$ across trials with every other voxel in the brain (Gazzaley et al., 2004; Rissman et al., 2004).

Analysis of each whole-brain network map used ANOVA that compared functional connectivity across memory performance to detect an interaction of two factors: recollection performance (correct vs incorrect) and condition (SHUT vs VD). ROIs were identified at a voxelwise threshold of $p<0.005$ and corrected for multiple comparisons ( $p$ corrected $<0.05$ ). Conjoined functional networks were revealed by masking network maps with each other (i.e., Fisher's procedure; Lazar et al., 2002) to identify clusters of overlapping voxels above the whole-brain clusterextent threshold of 12 voxels calculated with AlphaSim (Cox, 1996) for the whole-brain maps.

Correction for multiple comparisons. Voxel-based, pairwise $t$-tests (threshold of $p<0.001$, two-tailed) were carried out as group analyses across all 23 participants with participants as random effects. Monte Carlo simulations via the AplhaSim tool in AFNI (Cox, 1996) were then used to correct for multiple comparisons across a whole-brain volume based on gray matter voxels and to determine how large a cluster of nearest-neighbor voxels was needed to be statistically significant $(p<0.05)$.

Eye tracking during the memory test. Participants' eye movement and pupil diameter were recorded at a sampling rate of $120 \mathrm{~Hz}$ using a longrange infrared eye-tracking system (EyeTrac6 Long Range Optics System, Applied Science Laboratories). Gaze position was calculated by determining the distance between the center of the pupil and the corneal reflection with respect to a 9-point color calibration procedure. To correct for any calibration drift during the experiment, data for each stimulus presentation were normalized by resetting the origin to the observed fixation point during the final second of the ITI. Memory test trials when participants looked away from the center of the visual distractor image were excluded from analysis. Although participants' compliance with the task instructions was very high, data from one participant was excluded from further analysis because they looked away during the majority of their VD condition trials.

\section{Results}

\section{Behavioral experiment}

An estimation of $d^{\prime}$ for each participant, a measure that contrasts the hit rate for targets (i.e., both correct and incorrect responses for the number of items in the targets) with the false alarm rate for 


\begin{tabular}{|c|c|c|c|c|c|c|}
\hline & \multirow[b]{3}{*}{$d^{\prime}$} & \multicolumn{3}{|l|}{ Targets } & \multirow{2}{*}{\multicolumn{2}{|c|}{ Lures }} \\
\hline & & & Recollection & & & \\
\hline & & Recollected & Familiar & Forgotten & False alarms & Correct rejections \\
\hline \multicolumn{7}{|c|}{ Pilot study } \\
\hline Total & $2.10(0.09)$ & $56.1 \%(2.6)$ & $27.8 \%(1.7)$ & $16.0 \%(1.8)$ & $16.6 \%(2.7)$ & $83.4 \%(2.7)$ \\
\hline SHUT & $2.47(0.14)$ & $58.2 \%(2.9)$ & $27.0 \%(2.3)$ & $14.5 \%(1.8)$ & $12.4 \%(2.0)$ & $87.6 \%(2.0)$ \\
\hline GRAY & $2.09(0.14)$ & $57.1 \%(2.9)$ & $28.3 \%(2.0)$ & $14.5 \%(1.8)$ & $20.1 \%(3.2)$ & $79.9 \%(3.2)$ \\
\hline VD & $1.97(0.10)$ & $53.1 \%(2.6)$ & $27.6 \%(1.7)$ & $19.1 \%(2.0)$ & $17.3 \%(2.9)$ & $82.7 \%(2.9)$ \\
\hline \multicolumn{7}{|c|}{ fMRI experiment } \\
\hline Total & $1.96(0.10)$ & $51.0 \%(1.9)$ & $38.2 \%(1.6)$ & $10.8 \%(1.8)$ & $30.0 \%(3.1)$ & $70.0 \%(3.1)$ \\
\hline SHUT & $2.01(0.12)$ & $53.9 \%(2.0)$ & $35.7 \%(1.9)$ & $10.4 \%(1.8)$ & $28.5 \%(3.6)$ & $71.5 \%(3.6)$ \\
\hline GRAY & $1.79(0.14)$ & $49.3 \%(2.5)$ & $39.5 \%(2.1)$ & $11.2 \%(1.8)$ & $35.2 \%(4.2)$ & $64.8 \%(4.2)$ \\
\hline VD & $2.08(0.14)$ & $50.0 \%(2.3)$ & $39.4 \%(2.0)$ & $10.6 \%(2.0)$ & $26.3 \%(3.8)$ & $73.7 \%(3.8)$ \\
\hline
\end{tabular}

Top, Summary of statistics describing the responses to 168 targets and 36 lures that were presented during the test session of a pilot behavioral study. The mean performance for 27 participants is given in each of the three visual conditions (SEM). Bottom, Summary of statistics describing the behavioral responses from participants in the fMRI experiment. The mean performance for the 23 participants is given in each of the three visual conditions (SEM).

\section{Recollection}

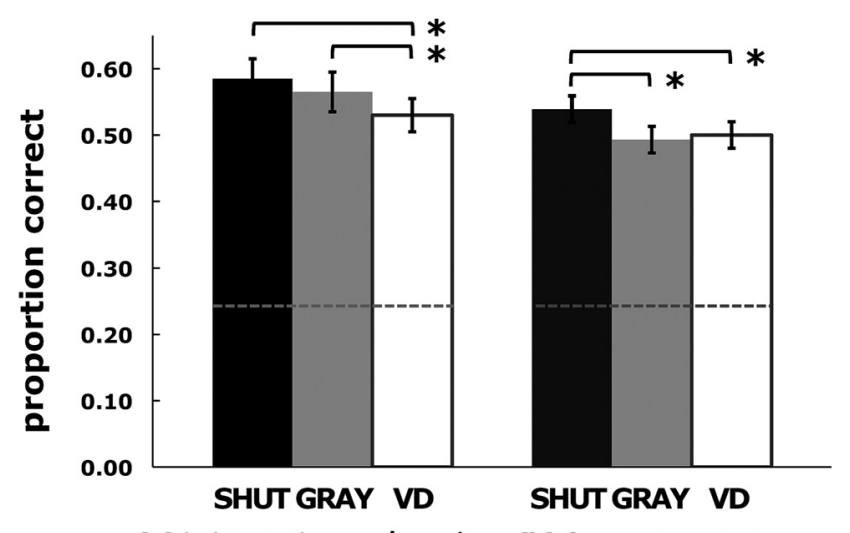

(a) behavioral experiment

(b) FMRI experiment

Figure 2. Recall success. Results for the behavioral experiment $(\boldsymbol{a})$ and the fMRl experiment (b) show the mean proportion of the targets given correct recollection responses in the SHUT, GRAY, and VD conditions. Error bars represent the SEM, ${ }^{*} p<0.05$.

lures (Macmillan and Creelman, 2005), was used to index overall memory performance for each of the test conditions (mean overall $\left.d^{\prime}=2.10 \pm 0.09\right)$. A comparison across test conditions showed a main effect of condition $\left(F_{(2,52)}=8.16, p<0.001\right)$, such that $d^{\prime}$ was greater for SHUT than both GRAY and VD (Table 1, top). Further evaluation of the recollection accuracy of the recall responses for targets (i.e., correct responses for the number of items in the targets) revealed a main effect of condition. Post hoc paired $t$ tests showed that recollection during VD was significantly reduced compared with both SHUT $(p<$ $0.001)$ and GRAY $(p<0.05)$ (Fig. 2). This finding was in contrast to the results for the targets recognized as previously viewed but given incorrect recall responses for the number of items, which showed no effect of visual distraction $\left(F_{(2,52)}=0.26\right)$. For the lures, a main effect of condition was evident, such that false alarms were fewer for SHUT than for GRAY or VD $\left(F_{(2,52)}=\right.$ $3.518, p<0.05$ ), with no difference between GRAY and VD.

\section{fMRI experiment}

\section{Behavioral results}

Overall memory performance (mean $d^{\prime}=1.96 \pm 0.10$ ) was not different across the test conditions $\left(F_{(2,44)}=1.70\right.$; Table 1 , bottom). However, an evaluation of the recollection accuracy of the recall responses for the targets revealed a main effect of condition
$\left(F_{(2,44)}=4.50, p<0.05\right)$, such that the proportion of targets given correct recollection responses was greater for SHUT than for GRAY or VD (both pairwise comparisons, $p<0.05$ ), with no difference between GRAY and VD. The comparison of the results for the targets that were recognized but given incorrect recall responses did not find an effect of condition $\left(F_{(2,44)}=2.23\right)$, although a pairwise comparison showed that VD incorrect was greater than SHUT incorrect $(p<0.05)$. There were no effects of condition for forgotten items, false alarms, or correct rejections.

The behavioral results from the fMRI experiment revealed that the mere act of having one's eyes open diminished recollection accuracy and replicated the findings from the behavioral study that VD disrupts recollection relative to SHUT (Fig. 2). However, the results for the gray screen condition differed between the two experiments, such that accuracy on GRAY did not differ from SHUT in the behavioral experiment, while accuracy on GRAY did not differ from VD in the fMRI experiment. We suspect that this difference may be the result of additional visual distraction in the GRAY condition in the fMRI experiment caused by the visible framework of the fMRI head-coil. Subsequent analysis of fMRI data was guided by converging results from the behavioral and fMRI experiments that suggested the contrast between SHUT and VD was the critical comparison to explore the impact of visual distraction on recollection.

\section{fMRI results}

Functional localizer. Object-selective regions in the visual association cortex were identified using an independent functional localizer task by contrasting activity associated with viewing objects versus viewing scrambled objects (voxelwise threshold, $p<$ 0.001 ). Activity was increased in several visual cortical areas bilaterally, and to the greatest magnitude in an area of the left lateral occipital cortex (LOC) (cluster size of 23 voxels surrounding the peak voxel; supplemental Figure S1, available at www.jneurosci. org as supplemental material), consistent with previous studies that have identified object-selective areas (Schwarzlose et al., 2008). This region served as a functional ROI associated with the selective visual representation of common objects, such as the target stimuli used in the study session.

Scene-selective regions in the visual association cortex were identified by contrasting activity associated with viewing outdoor scenes versus viewing objects (voxelwise threshold, $p<0.001$ ). Activity was increased in several visual areas, bilaterally, and to the greatest magnitude in a posterior area of the right PPA (cluster size of 36 voxels surrounding the peak voxel; supplemental Figure S1, available at www.jneurosci.org as supplemental mate- 


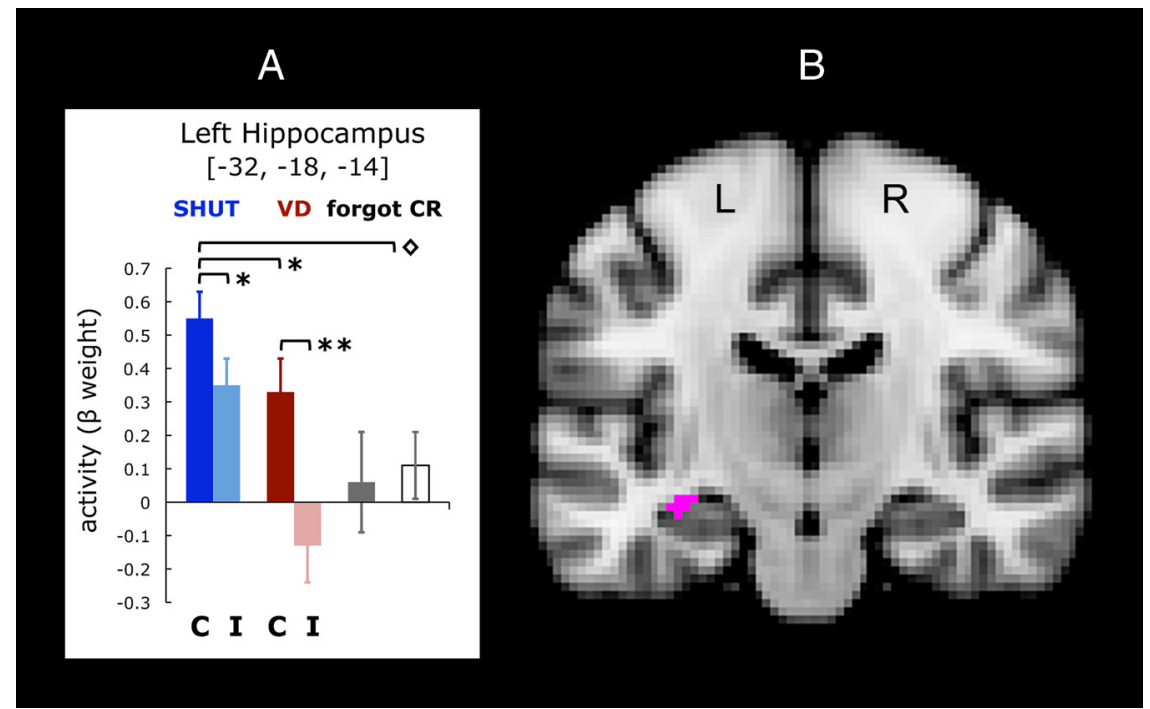

Figure 3. Univariate results. Activity in the left hippocampus increased selectively in association with recollection. The ANOVA of whole-brain activity that compared factors for SHUT correct, SHUT incorrect, VD correct, VD incorrect, forgot and correct rejections (CR) identified this functional region of interest (voxelwise threshold, $p<0.001$; $p$ corrected $<0.05$ ) that evidenced an interaction of memory performance and conditions. $A$, Mean values were extracted for the $\beta$-activity associated with each of the six categories of responses, and the analysis revealed that activity associated with recollection was negatively impacted by interference from visual distraction, such that VD correct was diminished relative to SHUT correct. On the $x$-axis, C indicates recognition when recall was correct, and I indicates recognition with incorrect recall. Error bars represent the SEM, ${ }^{*} p<0.05,{ }^{* *} p<0.001 ; \diamond$ indicates the differences for SHUT correct between forgot and $C R, p<0.05$. $B$, The left hippocampus cluster ( 25 voxels) is shown on a coronal view of the MNI brain template.

rial), also consistent with previous reports (Schwarzlose et al., 2008). The PPA served as a functional ROI associated with the visual representation of complicated scenes, such as the visual distractors presented in the VD condition in the test session.

Univariate analysis. The first step in the fMRI analysis was to identify memory-related brain regions that supported recognition independent of condition. These regions were found by performing a repeated-measures ANOVA of whole-brain activity associated with three categories: hits (i.e., targets given responses with correct or incorrect count), misses (i.e., targets endorsed with a response of "new") and correct rejections (i.e., lures endorsed as "new"). Analysis of regions that exhibited significant main effects in this ANOVA (voxelwise threshold, $p<0.001 ; p$ corrected $<0.05$ ) identified areas bilaterally in the PFC, MTL, parietal lobe and visual association cortex where activity increased in association with hits relative to misses, which were the same regions as for the comparison of hits relative to correct rejections (i.e., "old/new" effects; supplemental Table S1, available at www.jneurosci.org as supplemental material).

Data from these memory-related ROIs were further interrogated with a second ANOVA to identify regions that exhibited a differential response across recollection performance (correct vs incorrect) and condition (SHUT vs VD). Based on the behavioral data and our initial hypothesis that recollection of the details of studied images is impaired when visual distraction is present during the memory test, we probed each ROI that exhibited a significant interaction of recollection performance $\times$ condition. This revealed that only a single region, located in the left hippocampus, demonstrated the neural signature of a distraction effect: SHUT correct different from VD correct (Fig. 3; 25 voxels, $p$ corrected $<0.05 ; F_{(1,22)}=5.28$, interaction $\left.p<0.03\right)$. Significantly, the univariate results revealed that left hippocampal activity associated with recollection was negatively impacted by interference from visual distraction, such that VD correct was diminished relative to SHUT correct (pairwise comparison, $\left.t_{(22)}=2.20, p<0.05\right)$. Furthermore, although VD correct was significantly greater than VD incorrect $\left(t_{(22)}=3.80\right.$, $p<0.001)$, VD correct was not different compared with forgotten items or correct rejections (as it was for SHUT correct).

We next conducted a univariate analysis using the left LOC, object-selective ROI identified with the independent functional localizer task (supplemental Fig. S1, available at www.jneurosci.org as supplemental material). The results showed that activity associated with SHUT correct was greater in this region relative to the fixation baseline, despite eyes being closed, and was greater than that associated with SHUT incorrect (pairwise comparison, $t_{(22)}=$ $2.83, p<0.01)$. This pattern of activity in a stimulus-selective area of the visual association cortex in the absence of visual stimulation suggests that this region subserved the representation of target objects during successful recollection. This result offers strong evidence of visual imagery during recollection because, unique to this study, the participants' eyes were closed, and so increased activity in the left LOC could not have been associated with processing external visual stimuli. Indeed, all 23 participants indicated during postexperiment interviews that they used visual imagery in their strategy to answer the recall cues.

Activity in the left LOC increased in the VD condition relative to SHUT, with no difference between VD correct and VD incorrect. This pattern may be interpreted as evidence that distractionrelated interference due to bottom-up processing of external stimuli exacted a toll on visual imagery by engaging the same neural substrate. However, we are cautious about this interpretation because the presence of visual information in VD may have saturated the LOC response, preventing a sensitive assessment of the impact on visual imagery.

Functional connectivity analysis. To identify neural networks that subserved recollection and evaluate their susceptibility to interference effects during visual distraction, a whole-brain, $\beta$-series correlation analysis was performed to assess functional connectivity between regions across memory conditions (Gazzaley et al., 2004; Rissman et al., 2004). The left hippocampus ROI was used as a seed region because it was identified in the univariate analysis to subserve recollection in the SHUT condition, and its activity profile was disrupted in VD. The left LOC object-selective ROI identified using the independent functional localizer task was selected as a seed region to evaluate networks that support visual imagery during recollection. Using these two seed regions, whole-brain network maps were generated and an ANOVA was performed for each seed network that compared functional connectivity across recollection performance (correct vs incorrect) and condition (SHUT vs VD).

Analysis of the left hippocampus network identified two regions that evidenced an interaction in functional connectivity for recollection $\times$ condition. This included a region in the left inferior frontal gyrus (IFG) (40 voxels, $p$ corrected $<0.05 ; F_{(1,22)}=$ 4.92 , interaction $p<0.05)$ and a region in the left supramarginal gyrus $(\mathrm{SMG})\left(16\right.$ voxels, $p$ corrected $<0.05 ; F_{(1,22)}=5.76$, interaction $p<0.05)$. Significantly, the results showed that functional 


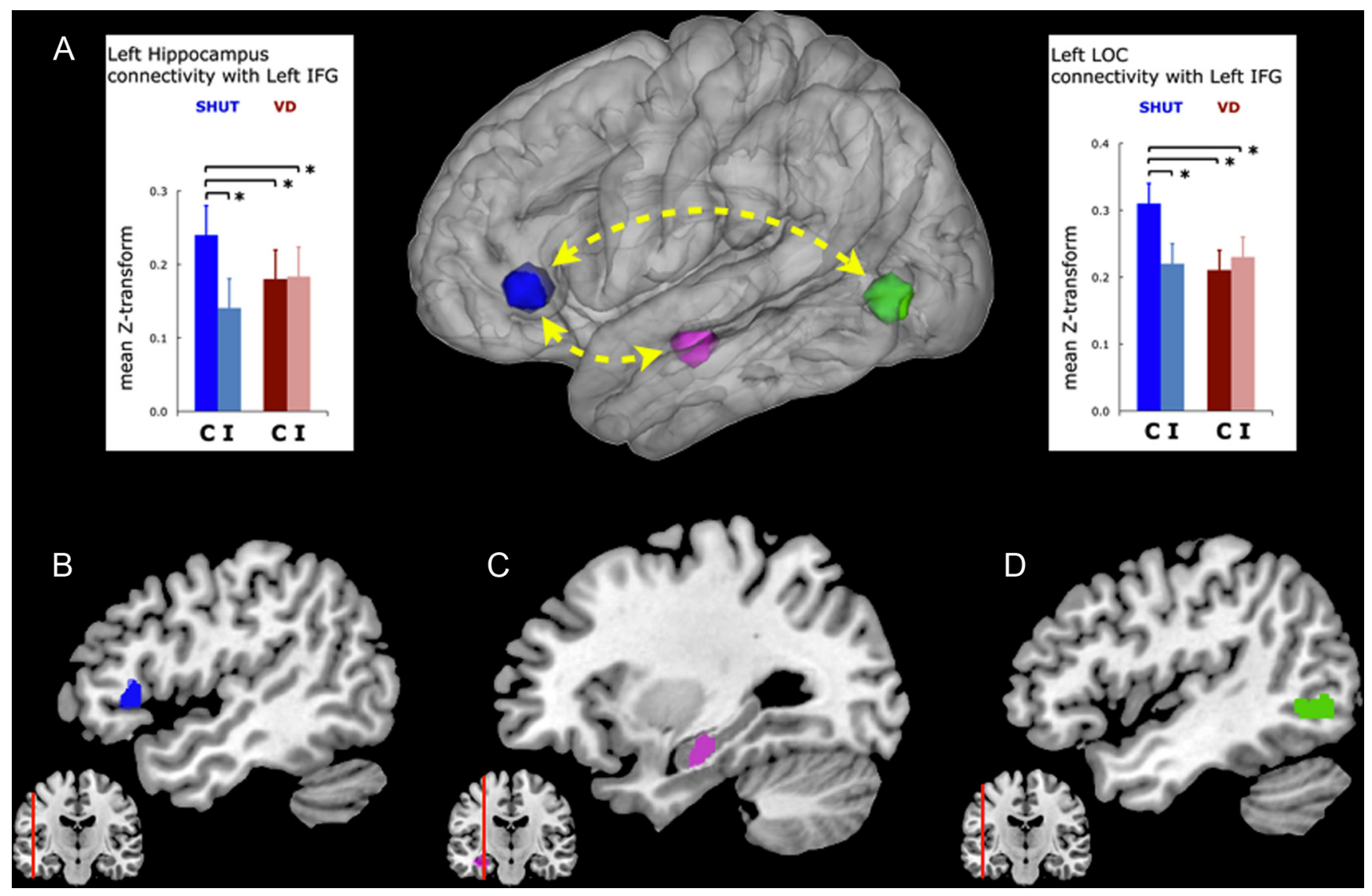

Figure 4. Functional connectivity in a recollection network. $A$, Aschematic of the recollection network is shown, and functional connectivity between the regions is plotted as the mean $z$-score transformation of the $\beta$-series correlations for correct (C) and incorrect (I) responses to targets during the SHUT and VD conditions. $\boldsymbol{B}-\boldsymbol{D}$, The network regions include the left IFG (B, blue cluster), the left hippocampus ( $\boldsymbol{C}$, violet cluster), and the left LOC ( $\boldsymbol{D}$, green cluster). The analysis revealed that functional connectivity between the IFG and LOC (right graph), which supported recollection during SHUT, was disrupted during VD. Error bars represent the SEM, ${ }^{*} p<0.05$.

connectivity with the left hippocampus was greater for both regions during SHUT correct compared with VD correct. The analysis of the left LOC network identified one region that demonstrated an interaction in functional connectivity: a region in the left IFG (26 voxels, $p$ corrected $<0.05 ; F_{(1,22)}=5.13$, interaction $\left.p<0.05\right)$. Functional connectivity between the left IFG region and the left LOC was also greater during SHUT correct than VD correct. Using maps generated by ANOVA in the analyzes above, a conjunction analysis was accomplished by masking the left hippocampus and left LOC network maps with each other (i.e., Fisher's procedure; Lazar et al., 2002) and revealed a single region in common that displayed an interaction: a region in the left IFG ( 26 voxels, $p$ corrected $<0.05$ ), approximately pars triangularis (BA45) (Fig. 4).

Next, we evaluated the impact of visual distraction on these network connections via pairwise comparisons of functional connectivity measures during SHUT correct and VD correct (Fig. 4). Analysis revealed that connectivity significantly decreased with the IFG in association with VD correct for both comparisons (left hippocampus, $t_{(22)}=2.32, p<0.05$; left LOC, $\left.t_{(22)}=2.92, p<0.05\right)$, and, critically, no longer supported successful recollection (i.e., no difference between VD correct and VD incorrect). Moreover, an across-participant regression analysis showed that the change in network connectivity between SHUT correct and VD correct was correlated between an index of left IFG to left hippocampus connectivity and an index of left IFG to left LOC connectivity $(r=0.42, p<$ 0.05 ; Fig. 5). This revealed that when IFG network connectivity was decreased with the LOC, it was also decreased with the hippocam-

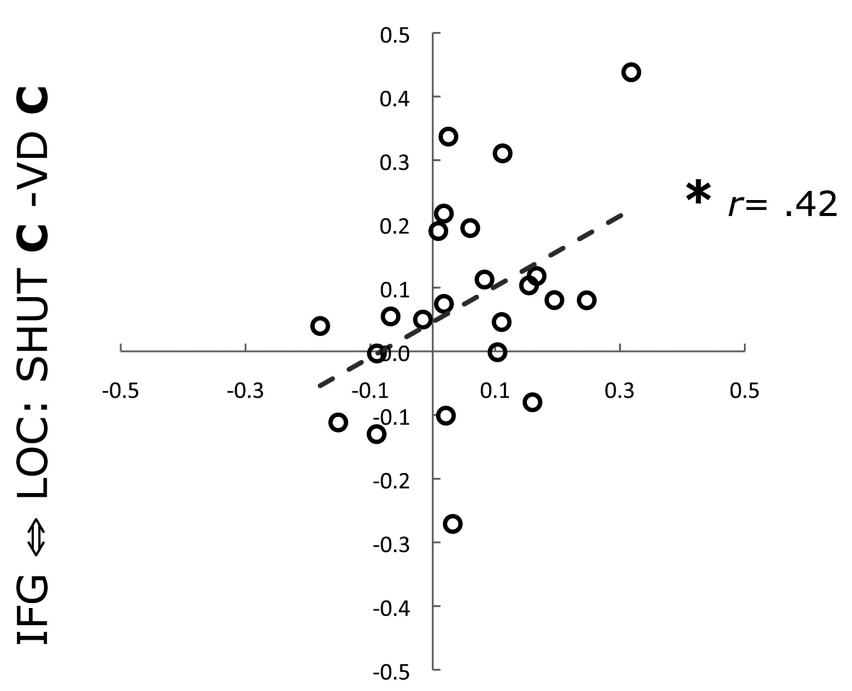

\section{IFG $\Leftrightarrow$ Hipp. : SHUT C -VD C}

Figure 5. Changes in left IFG functional connectivity are correlated between left hippocampus and left LOC. A scatter plot shows the values for each participant in a regression analysis of functional connectivity between the left IFG and the left hippocampus ROIs ( $x$-axis, SHUT correct versus VD correct) and the left IFG and the left LOC ROIs ( $y$-axis, SHUT correct versus VD correct). The analysis revealed that reduced left IFG connectivity with the left $L O C$ was correlated with reduced connectivity with the left hippocampus. Trend lines show the slope of significant correlations. ${ }^{*} p<0.05$. 


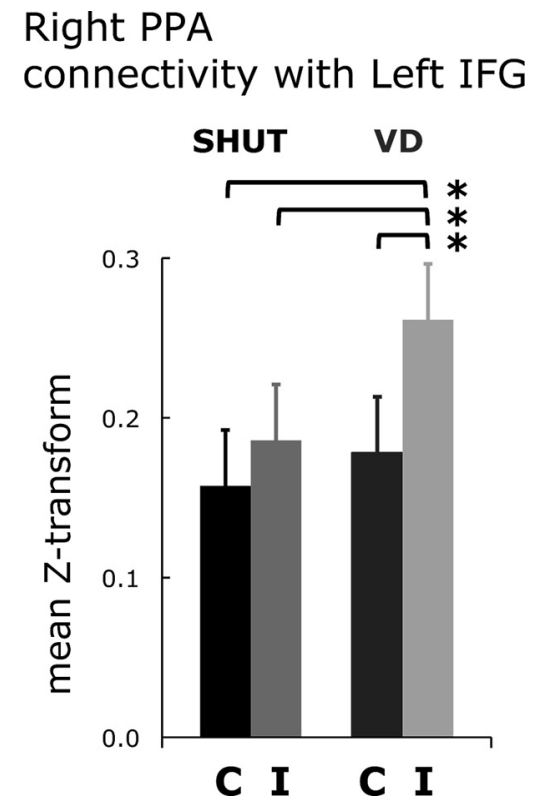

Figure 6. Functional connectivity between right PPA and left IFG is greater during VD incorrect than VD correct. Functional connectivity between the left IFG region in the recollection network (Fig. 4) and a scene-selective region in the right PPA is plotted as the mean z-score transformation of the $\beta$-series correlations for each of four categories for responses to the targets. Error bars represent the SEM, and ${ }^{*} p<0.05$. tention associated with processing the irrelevant stimuli during VD. To assess this hypothesis, we performed a $\beta$-series correlation analysis using the scene-selective PPA (identified with the independent localizer task) as a seed and the left IFG as an ROI. The analysis revealed that functional connectivity between the left IFG and the PPA was greater during VD incorrect than VD correct (Fig. 6).

To summarize, the functional connectivity results reveal that when visual distraction negatively impacted recollection (i.e., VD correct), left IFG connectivity decreased with the left hippocampus and the left LOC, and when recollection failed (i.e., VD incorrect), connectivity increased with the right PPA, a region where irrelevant visual stimuli are maximally represented.

\section{Subgroup analysis}

Further assessment of the impact of visual distraction on recollection was conducted by subdividing the participants into two groups based on the influence distraction had on recollection performance. Participants whose recollection accuracy was reduced during VD relative to SHUT were classified as Distracted, and participants whose accuracy was equal or better in VD relative to SHUT were categorized as Non-Distracted (Fig. 7A). For the Distracted subgroup $(n=13)$, mean SHUT correct $=57 \pm$ $10 \%$ and mean VD correct $=45 \pm 9 \%$. For the Non-Distracted subgroup $(n=10)$, mean SHUT correct $=50 \pm 9 \%$ and mean VD correct $=54 \pm 7 \%$. Unequal-variance, two-tailed t-tests found that SHUT correct performance was not different between the two groups, whereas VD correct performance was significantly reduced for the Distracted participants compared with the Non-Distracted participants $\left(t_{(22)}=2.18, p<0.05\right)$, revealing that the distinction between Distracted and Non-Distracted participants was driven by the impact of VD and not differences in performance when eyes were shut.

For the Non-Distracted participants, activity in the left hippocampus associated with SHUT correct was not different from VD correct. For the Distracted participants, however, left hippocampal activity was reduced during VD correct, relative to SHUT correct $\left(t_{(12)}=2.21, p<0.05\right)$, and VD correct activity was not different from the level associated with the forgotten items (Fig. 7B). This was the same pattern observed for the whole cohort of participants (Fig. 3). The difference in the pattern of left hippocampal activity between subgroups reveals that the impact of visual distraction on hippocampal activity was not uniform across our sample, but was a characteristic of the Distracted subgroup. The finding thus supports the relationship between a decline in hippocampal activity in pus. This result suggests that diminished recollection accuracy in the setting of visual distraction is mediated by a disruption of a network involving the IFG, the hippocampus and the visual association cortex that supports retrieval of contextual details based on visual imagery.

Recent work has shown that the IFG serves at the junction of cognitive control functions that mediate perception, attention, working memory and LTM systems (Badre and Wagner, 2007; Ranganath et al., 2007; Chong et al., 2008; Nee and Jonides, 2008). We thus hypothesized that a source of interference with the left IFG network's role in visual imagery and recollection may have been concurrent involvement in bottom-up driven visual at- the presence of visual distraction and a decline in recollection performance.

\section{Discussion}

In the current study, we investigated the impact irrelevant visual information has on LTM. Participant performance in both the behavioral and fMRI experiments revealed that irrelevant visual stimuli presented during a memory test diminished recollection performance. This finding suggests that there is a critical role for cognitive control processes in minimizing the disruptive influence of irrelevant external information during recollection. The 
failure to inhibit the processing of distractions has also been shown to diminish accuracy in perception and visual working memory (WM) (Lavie et al., 2004; Gazzaley et al., 2005a, 2008; Zanto and Gazzaley, 2009; Clapp et al., 2010). The current results extend this concept to the disruption of episodic memory retrieval.

\section{Distraction interrupts recollection accuracy}

Recent studies have distinguished between the impact of interference from distraction (entirely irrelevant information) and interruption (relevant information for a secondary task) on WM, and revealed that distinct neural mechanisms underlie these two types of interference (Clapp et al., 2010), as well as the presence of differential effects in aging (Clapp and Gazzaley, 2010). The current study specifically explored the influence of distraction-related interference on recollection in young adults, as the visual stimuli in the VD condition were entirely irrelevant (i.e., participants were explicitly instructed to direct their undivided attention to the goal of responding to the memory test). Our findings of a decrement in recollection in the setting of distraction parallel the documented impact by interruption (dual-tasking) on LTM (Jacoby, 1991; Troyer et al., 1999; Fernandes and Moscovitch, 2000; Fernandes et al., 2006), but likely involve distinct neural mechanisms.

This behavioral finding raises the potential for two, nonmutually exclusive, neural mechanisms that may underlie the impact of distraction on recollection. First, bottom-up, visual processing of external information may result in a decrease in the fidelity of internal representations of memoranda generated via visual imagery during the retrieval period, because both types of representations rely on overlapping regions of visual cortices. Second, because attentional resources are limited (Pashler and Shiu, 1999), top-down effort required to retrieve memories when cued may suffer when incidental attention to the irrelevant visual information diverts resources away from LTM goals. Although this diversion would be driven by bottom-up attention, since there were no top-down goals to attend to the visual stimuli, excessive demands on networks in common across these processes may result in memory performance impairment.

\section{Visual distraction impacts hippocampal function}

Whole-brain univariate analysis of fMRI data revealed an interaction of recollection performance $\times$ condition in a region of the left hippocampus, such that the presence of visual distraction generated a pattern indicative of distraction-related interference with recollection (i.e., Shut correct $>$ VD correct). Previous work has established a role for the hippocampus in support of episodic memory (Brown and Aggleton, 2001; Eichenbaum et al., 2007). Our study reveals for the first time that recollection performance declines in the presence of irrelevant external information and that this decline is associated with disrupted hippocampal function.

\section{Interference with the recollection network}

Although network connectivity that subserves visual imagery (Mechelli et al., 2004) and episodic retrieval (Burianova et al., 2010) have been examined independently, a common neural network that supports visual imagery during recollection has not been well defined. Key findings from the current study were that functional connectivity between the left IFG and both the left hippocampus and LOC was associated with successful performance in the SHUT condition and that this network connectivity was disrupted in the presence of irrelevant visual information in the VD condition (i.e., significantly reduced for VD correct compared with SHUT correct, and no longer different between VD correct and VD incorrect) (Fig. 4). Prior research has found that the left IFG (BA45) subserves the selection of contextual details during successful recollection (Zhang et al., 2004; Dobbins and Wagner, 2005; Law et al., 2005; Wais, 2010; Wais et al., 2010). This process is sometimes described as memorial selection to distinguish it from other attentional control processes mediated by IFG regions during perceptual selection (Corbetta et al., 2008; Nee and Jonides, 2009). Moreover, the left IFG has also been identified in studies that explored the reinstatement of cortical encoding activity during later recognition tests (Wheeler and Buckner, 2004; Johnson et al., 2009). The mutual functional connectivity of a left IFG region with an object-selective region involved in visual imagery and a memory region critical for recollection suggests that the left IFG may serve as a source of cognitive control in a network that supports the selection of contextual details via visual imagery during recollection. Interference with memorial selection would disrupt retrieval of the contextual information necessary for successful recollection.

The influence of visual distraction was evident not only in recollection network connectivity, but also in increased connectivity between the left IFG and the scene-selective region in the right PPA during VD incorrect responses (relative to VD correct) (Fig. 6). Although there was not a significant inverse correlation between changes in connectivity in the IFG recollection network and changes in connectivity between the IFG and PPA, the shifting pattern in left IFG connectivity does suggest that disruption of the recollection network may be mediated by bottom-up driven attention. Although a recent view has been that the ventral attention system involves IFG control processes that do not mediate reorienting attention to irrelevant stimuli (Kincade et al., 2005; Corbetta et al., 2008), the influence of the distracting scenes in the current study may have been substantially greater than the simple visual distractors used in prior work. This would be consistent with the second mechanism we propose for the disruptive effect of distraction, i.e., interference with top-down control networks.

It is also possible that control processes subserved by the left IFG that guide the selection of contextual details during LTM (Badre and Wagner, 2007) were recruited to modulate retroactive interference from irrelevant perceptual information (Braver et al., 2007). Although recent work suggests that the left IFG is associated with suppressing task-irrelevant actions (Chong et al., 2008) and with interference resolution during semantic retrieval (Nelson et al., 2009), the question remains as to whether increased left IFG activity contributes to the resolution of interference from irrelevant visual information (Nee and Jonides, 2009). Thus, another explanation for increased functional connectivity between the left IFG and the scene-selective region in the right PPA during VD incorrect is that it reflects an overwhelming influence of bottom-up processing of irrelevant exogenous information over control processes that protect against interference with memorial selection. The additional demand on capacitylimited control processes driven by distractor salience may have shifted left IFG function away from memorial selection.

Another region that demonstrated a recollection performance $X$ condition interaction in functional connectivity with the left hippocampus seed was the left SMG (approximately BA40). This parietal region has been implicated in the experience of confidence about LTM (Vilberg and Rugg, 2009), the memory retrieval mode of the default network (DN) (Buckner et al., 2008) and a broader characterization of the dorsal attention network involved in the maintenance of internally generated information (Corbetta et al., 2008). Functional connectivity between the left SMG and left hippocampus may have guided participants' attention to the retrieval goal but, because the SMG network not did 
not also exhibit an interaction with the left LOC, the results do not implicate the SMG in the disruption of the retrieval of contextual details based on visual imagery.

\section{Recollection involves visual imagery}

The fMRI data obtained during the SHUT condition revealed strong evidence that recollection involved visual imagery. Correct recollection responses were associated with greater activity in the left LOC, relative to incorrect responses and resting baseline. Because the participants' eyes were shut during these trials, we interpret the increased activity in the LOC during retrieval as evidence for the reinstatement of encoded representations for the target objects, or visual imagery. This is consistent with results from previous fMRI studies that have shown reinstatement of activity associated with encoding visual stimuli when recognition was successful (Wheeler and Buckner, 2004; Johnson and Rugg, 2007; Johnson et al., 2009). However, the conclusions from prior research were limited to interpretations about subjective recollection and by the processing of visual memory cues concurrent with reinstatement of visual imagery engaged for the studied items. Our approach addressed these limitations by probing the objective recollection of contextual details of the memoranda when the participant's eyes were shut so that no external information was being processed in the visual association cortex during the memory test.

\section{Conclusion}

The results of the current study reveal the sensitivity of our longterm memory system to disruption by the presence of irrelevant environmental stimuli, such that the mere act of having our eyes open decreases the accuracy of memory retrieval. Furthermore, a memory network involving the left hippocampus, prefrontal cortex, and visual association cortex, which supports visual imagery and successful recollection when our eyes are closed, is disrupted by external distraction. This impact on performance and functional connectivity are likely mediated by capacity limitations in frontal control processes. A focus of future work will be to assess whether there is an exacerbation of this phenomenon associated with cognitive aging, as has been revealed for diminished WM performance in older adults (Gazzaley et al., 2005b, 2008).

\section{References}

Anderson N (2001) Empirical direction in design and analysis. Mahwah, NJ: Erlbaum.

Atkinson R, Juola J (1973) Factors influencing the speed and accuracy of word recognition. In: Attention and performance (Kornblum S, ed), pp 583-612. New York: Academic.

Badre D, Wagner AD (2007) Left ventrolateral prefrontal cortex and the cognitive control of memory. Neuropsychologia 45:2883-2901.

Blumenfeld RS, Ranganath C (2006) Dorsolateral prefrontal cortex promotes long-term memory formation through its role in working memory organization. J Neurosci 26:916-925.

Braver T, Gray J, Burgess G (2007) Explaining the many varieties of working memory variation: dual mechanisms of cognitive control. In: Variation in working memory (Conway A, Jarrold C, Kane M, Miyake A, Towse J, eds), pp 76-106. New York: Oxford UP.

Brown MW, Aggleton JP (2001) Recognition memory: what are the roles of the perirhinal cortex and hippocampus? Nat Rev Neurosci 2:51-61.

Buckner RL, Wheeler ME (2001) The cognitive neuroscience of remembering. Nat Rev Neurosci 2:624-634.

Buckner RL, Andrews-Hanna JR, Schacter DL (2008) The brain's default network: anatomy, function, and relevance to disease. Ann N Y Acad Sci 1124:1-38.

Burianova H, McIntosh AR, Grady CL (2010) A common functional brain network for autobiographical, episodic, and semantic memory retrieval. Neuroimage 49:865-874
Chong TT, Williams MA, Cunnington R, Mattingly JB (2008) Selective attention modulates inferior frontal gyrus activity during activity observation. Neuroimage 40:298-307.

Clapp WC, Gazzaley A (2010) Distinct mechanisms for the impact of distraction and interruption on working memory in normal aging. Neurobiol Aging. Advance online publication. Retrieved February 16, 2010 doi:10.1016/j.neurobiolaging.2010.01.012.

Clapp WC, Rubens MT, Gazzaley A (2010) Mechanisms of working memory disruption by external interference. Cereb Cortex 20:859-872.

Corbetta M, Patel G, Shulman GL (2008) The reorienting system of the human brain: from environment to theory of mind. Neuron 58:306-324.

Cox RW (1996) AFNI: Software for analysis and visualization of functional magnetic resonance neuroimages. Comput Biomed Res 29:162-173.

de Fockert JW, Rees G, Frith CD, Lavie N (2001) The role of working memory in visual selective attention. Science 291:1803-1806.

Dobbins IG, Wagner AD (2005) Domain-general and domain-sensitive prefrontal mechanisms for recollecting events and detecting novelty. Cereb Cortex 15:1768-1778.

Eichenbaum H, Yonelinas AP, Ranganath C (2007) The medial temporal lobe and recognition memory. Annu Rev Neurosci 30:123-152.

Fernandes MA, Moscovitch M (2000) Divided attention and memory: evidence of substantial interference effects at retrieval and encoding. J Exp Psychol Gen 129:155-176.

Fernandes MA, Pacurar A, Moscovitch M, Grady C (2006) Neural correlates of auditory recognition under full and divided attention in younger and older adults. Neuropsychologia 44:2452-2464.

Gazzaley A, Rissman J, D’Esposito M (2004) Functional connectivity during working memory maintenance. Cogn Affect Behav Neurosci 4:580-599.

Gazzaley A, Cooney JW, McEvoy K, Knight RT, D’Esposito M (2005a) Topdown enhancement and suppression of the magnitude and speed of neural activity. J Cogn Neurosci 17:507-517.

Gazzaley A, Cooney JW, Rissman J, D’Esposito M (2005b) Top-down suppression deficit underlies working memory impairment in normal aging. Nat Neurosci 8:1298-1300.

Gazzaley A, Clapp W, Kelley J, McEvoy K, Knight RT, D’Esposito M (2008) Age-related top-down suppression deficit in the early stages of cortical visual memory processing. Proc Natl Acad Sci U S A 105:13122-13126.

Glenberg AM, Schroeder JL, Robertson DA (1998) Averting the gaze disengages the environment and facilitates remembering. Mem Cognit 26:651-658.

Ishai A, Ungerleider LG, Haxby JV (2000) Distributed neural systems for the generation of visual images. Neuron 28:979-990.

Jacoby L (1991) A process dissociation framework: separating automatic from intentional uses of memory. J Mem Lang 30:513-541.

James W (1890) The principles of psychology. New York: Holt.

Johnson JD, Rugg MD (2007) Recollection and the reinstatement of encoding-related cortical activity. Cereb Cortex 17:2507-2515.

Johnson JD, McDuff SG, Rugg MD, Norman KA (2009) Recollection, familiarity, and cortical reinstatement: a multivoxel pattern analysis. Neuron 63:697-708.

Kahn I, Davachi L, Wagner AD (2004) Functional-neuroanatomic correlates of recollection: implications for models of recognition memory. J Neurosci 24:4172-4180.

Kincade JM, Abrams RA, Astafiev SV, Shulman GL, Corbetta M (2005) An event-related functional magnetic resonance imaging study of voluntary and stimulus-driven orienting of attention. J Neurosci 25:4593-4604.

Lavie N (2005) Distracted and confused? Selective attention under load. Trends Cogn Sci 9:75-82.

Lavie N, Hirst A, de Fockert JW, Viding E (2004) Load theory of selective attention and cognitive control. J Exp Psychol Gen 133:339-354.

Law JR, Flanery MA, Wirth S, Yanike M, Smith AC, Frank LM, Suzuki WA, Brown EN, Stark CE (2005) Functional magnetic resonance imaging activity during the gradual acquisition and expression of paired-associate memory. J Neurosci 25:5720-5729.

Lazar NA, Luna B, Sweeney JA, Eddy WF (2002) Combining brains: a survey of methods for statistical pooling of information. Neuroimage 16:538-550.

Macmillan N, Creelman C (2005) Detection theory: a user's guide, Ed 2. Mahwah, NJ: Erlbaum

Mandler G (1980) Recognizing: the judgment of previous occurrence. Psychol Rev 87:252-271.

Mechelli A, Price CJ, Friston KJ, Ishai A (2004) Where bottom-up meets 
top-down: neuronal interactions during perception and imagery. Cereb Cortex 14:1256-1265.

Nee DE, Jonides J (2008) Neural correlates of access to short-term memory. Proc Natl Acad Sci U S A 105:14228-14233.

Nee D, Jonides J (2009) Common and distinct neural correlates of perceptual and memorial selection. Neuroimage 25:963-975.

Nelson JK, Reuter-Lorenz PA, Persson J, Sylvester CY, Jonides J (2009) Mapping interference resolution across task domains: a shared control process in left inferior frontal gyrus. Brain Res 1256:92-100.

Pashler H, Shiu LP (1999) Do images involuntarily trigger search? A test of Pillsbury's hypothesis. Psychon Bull Rev 6:445-448.

Rainer G, Asaad WF, Miller EK (1998) Selective representation of relevant information by neurons in the primate prefrontal cortex. Nature 393:577-579.

Ranganath C, Cohen MX, Dam C, D'Esposito M (2004) Inferior temporal, prefrontal, and hippocampal contributions to visual working memory maintenance and associative memory retrieval. J Neurosci 24:3917-3925.

Ranganath C, Heller AS, Wilding EL (2007) Dissociable correlates of two classes of retrieval processing in prefrontal cortex. Neuroimage 35:1663-1673.

Rissman J, Gazzaley A, D’Esposito M (2004) Measuring functional connectivity during distinct stages of a cognitive task. Neuroimage 23:752-763.

Schwarzlose RF, Swisher JD, Dang S, Kanwisher N (2008) The distribution of category and location information across object-selective regions in human visual cortex. Proc Natl Acad Sci U S A 105:4447-4452.
Squire LR, Wixted JT, Clark RE (2007) Recognition memory and the medial temporal lobe: a new perspective. Nat Rev Neurosci 8:872-883.

Stokes M, Thompson R, Cusack R, Duncan J (2009) Top-down activation of shape-specific population codes in visual cortex during mental imagery. J Neurosci 29:1565-1572.

Troyer AK, Wincour G, Craik FI, Moscovitch M (1999) Source memory and divided attention: reciprocal costs to primary and secondary tasks. Neuropsychology 13:467-474.

Tulving E (1985) Memory and consciousness. Can J Psychol 26:1-12.

Vilberg KL, Rugg MD (2009) Functional significance of retrieval-related activity in lateral parietal cortex: evidence from fMRI and ERPs. Hum Brain Mapp 30:1490-1501.

Wais PE (2010) Hippocampal signals for strong memory when associative memory is available and when it is not. Hippocampus. Advance online publication. Retrieved December 9, 2009. doi:10.1002/hipo.20716.

Wais PE, Squire LR, Wixted JT (2010) In search of recollection and familiarity signals in the hippocampus. J Cogn Neurosci 22:109-123.

Wheeler ME, Buckner RL (2004) Functional-anatomic correlates of remembering and knowing. Neuroimage 21:1337-1349.

Zanto TP, Gazzaley A (2009) Neural suppression of irrelevant information underlies optimal working memory performance. J Neurosci 29:30593066.

Zhang JX, Feng CM, Fox PT, Gao JH, Tan LH (2004) Is left inferior frontal gyrus a general mechanism for selection? Neuroimage 23:596-603. 